\title{
Intestinal FoxO signaling is required to survive oral infection in Drosophila
}

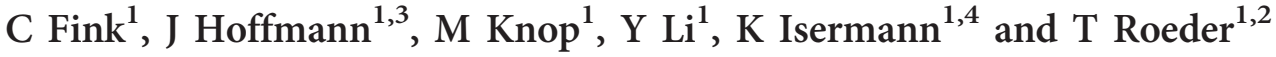

The intestinal immune system is tailored to fight pathogens effectively while tolerating the indigenous microbiota. Impairments of this homeostatic interaction may contribute to the etiology of various diseases including inflammatory bowel diseases. However, the molecular architecture underlying this complex regulatory interaction is not well understood. Here, we show that the fruit fly Drosophila melanogaster has a multilayered intestinal immune system that ensures strictly localized antimicrobial responses. Enterocytes, a major cell population of the intestine, produced antimicrobial peptides (AMPs) in a FoxO- but not NF-kB-dependent manner. Consequently, animals impaired in FoxO-mediated signaling had a significantly lowered resistance to intestinal infections; they were unable to increase the expression of AMP genes and males showed an increased bacterial load in response to an infection. Conventional innate immune signaling converging onto NF-кB activation was operative in only a few regions of the intestine, comprising the proventriculus, copper cells, and intestinal stem cells. Taken together, our results imply that danger-mediated as well as conventional innate immune signaling constitute modules that contribute to the fruit fly's intestinal immune system. We propose that this special architecture ensures localized and efficient antimicrobial responses against invasive pathogens while preserving the microbiota.

\section{INTRODUCTION}

The evolution of the intestinal immune system has been driven by the need to protect the organism from intestinal pathogens while supporting and preserving the complex and beneficial microbiota. ${ }^{1}$ In charge of the multifaceted interactions between the host and the environment is the intestinal epithelium. Epithelia are not only simple physical barriers but also sentinels that detect and fight pathogens. Moreover, they orchestrate most aspects of the mucosal immune response, presumably mediated through their innate immune signaling pathways. Besides its role in defense against pathogens, the intestinal epithelium shapes the composition of the indigenous microbiota by constant release of antimicrobial compounds. To reconcile these conflicting goals, the architecture of the intestinal immune system follows two major strategies, namely, stratification and compartmentalization of intestinal bacteria. ${ }^{1}$ Reducing direct contact between bacteria and epithelial cells (stratification) is of prime importance for the reduction of unwanted activation of the epithelial innate immune system. In mammals, the mucus layer, which is a relatively sterile barrier between epithelial cells and intestinal bacteria, is responsible for stratification. Mucus sterility is achieved by inclusion of antimicrobial compounds such as lectins or antimicrobial peptides (AMPs). ${ }^{2}$ Compartmentalization, on the other hand, is based on the effective removal of intestinal bacteria that penetrate the antimicrobial barrier. In addition to these general strategies, some endangered regions of the intestinal epithelium require special protection. Paneth cells guard crypts of the small intestine containing intestinal stem cells (ISCs). ${ }^{3}$ To achieve all these goals, the intestinal immune system has to be well balanced, which is achieved by dampening generalized immune responses that would compromise the microbiota while focusing these responses to highly endangered sites.

Prolonged impairment of the homeostatic balance between epithelial immunity and tolerance is believed to be responsible for a chain of events leading to chronic inflammatory responses, as manifested in inflammatory bowel diseases. ${ }^{4-6}$ Inflammatory bowel diseases are complex, polygenic diseases, with

${ }^{1}$ Department of Zoology, Molecular Physiology, Christian-Albrechts University Kiel, Kiel, Germany and ${ }^{2}$ Airway Research Center North, Member of the German Center for Lung Research (DZL), Germany. Correspondence: T Roeder (troeder@zoologie.uni-kiel.de)

${ }^{3}$ Current address: Max-Planck Institute for Biology of Ageing, Cologne, Germany.

${ }^{4}$ Current address: Eppendorf AG, Hamburg, Germany. 

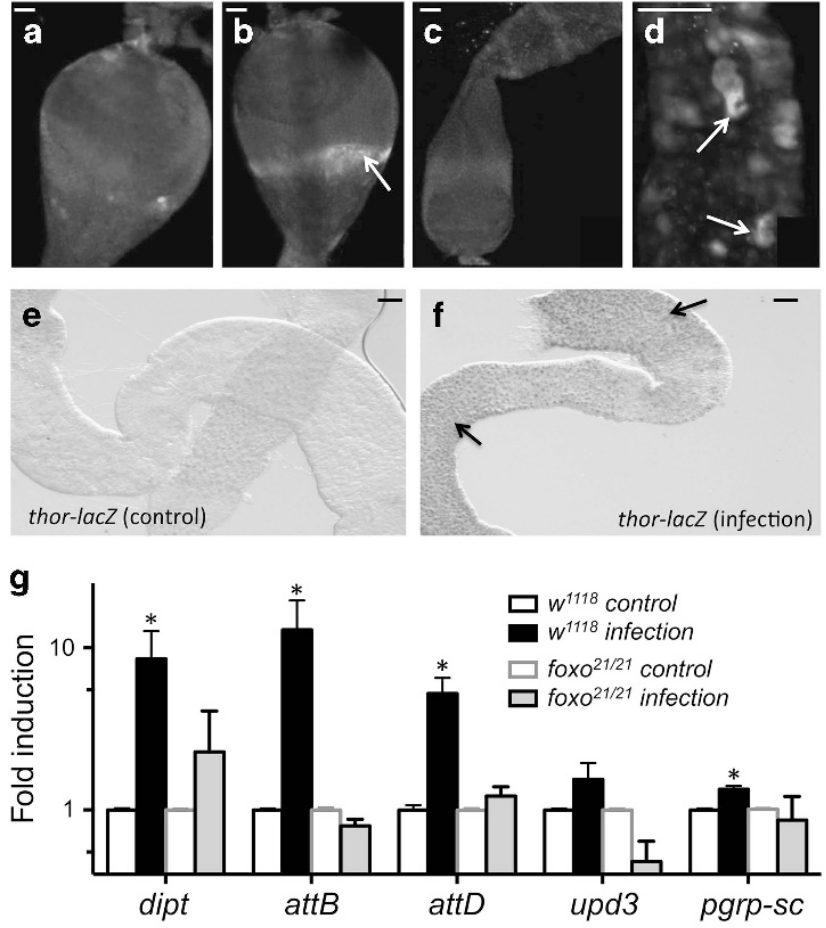

Figure 1 Oral infection with Serratia marcescens induces expression of antimicrobial peptide genes in different parts of the intestine and activates FoxO-dependent signaling. Shown are the responses of two transgenic lines indicating promotor activity of antimicrobial peptides (Defensin and Attacin) by concurrent expression of green fluorescent protein (GFP) in response to oral infection with $S$. marcescens. Controls fed with $5 \%$ sucrose did not show any signal in either the attacin- (a) or the defensinreporter lines $(\mathbf{c})$. Oral infection with Serratia induced attacin-associated expression primarily in parts of the proventriculus (b, arrow) and defensinassociated expression in various regions of the midgut (d, arrow). Oral infection with Serratia also induced activation of thorexpression, which is a canonical FoxO target gene, as visualized using a thor-lacZreporter strain (e and $\mathbf{f}$, arrow). Transcript levels of various immune-related genes in response to oral infection with Serratia (6 h post infection) was quantified using quantitative real-time PCR (qRT-PCR) with isolated intestines from infected and control $w^{1118}$ and foxo $o^{21 / 21}$ adults $(\mathbf{g}$; scale bars $(\mathbf{a}-\mathbf{f})=20 \mu \mathrm{m}$, in $\mathbf{g}$, mean values \pm s.e.m. of four biological replicates are shown, ${ }^{\star} P<0.05$, Mann-Whitney test).

environmental factors as well as genetic predisposition contributing to pathogenesis. Although the role of environmental factors is not well understood, ${ }^{7}$ a number of susceptibility genes have been identified. ${ }^{8}$ The first identified susceptibility gene for Morbus Crohn, NOD2, is an intracellular pattern recognition receptor (PPR), ${ }^{9}$ further highlighting the role of the innate immune system in maintaining a healthy epithelium.

Although our understanding of the major aspects of intestinal immunity increased substantially, very important issues, especially the control and architecture of the innate immune response of different cell types in the intestine or the mechanisms that dampen the response to protect the indigenous microbiota, remain elusive. Genetically tractable model organisms such as the fruit fly Drosophila melanogaster, which are equipped with a relatively simple innate immune system, allow us to experimentally address various unanswered questions. The fruit fly is able to cope with a great variety of different pathogens including bacteria, fungi, viruses, and eukaryotes, most of them trying to invade through the intestinal epithelium. ${ }^{10,11}$ Although the fly's intestinal epithelium is much more simply organized than the mammalian one, it is faced with exactly the same set of problems, apparently leading to similar approaches to solve them. Recently, some basic aspects of epithelial immune responses, especially of intestinal immune responses in Drosophila, have been characterized. The most important way to fight intestinal pathogens appears to be the generation of reactive oxygen species (ROS) via the dual oxidase (Duox). ${ }^{12,13}$ Conventional strategies of the innate immune system, including the NF-kB-dependent production of AMP, seem to function as back-up systems that become relevant in cases where ROS-resistant bacteria colonize the gut. ${ }^{14}$ Different mechanisms that dampen the immune response through microbe-associated microbial patterns (MAMPs) produced by the indigenous microbiota have been proposed. These include the release of amidases that inactivate petidoglycans $^{15,16}$ or inhibition of antimicrobial responses by the transcription factor caudal. ${ }^{17}$

In the current study, we tried to elucidate the architecture of the intestinal epithelium and understand the contribution of different cell types to the overall intestinal immune reaction. We found that FoxO signaling, which frequently mediates danger/stress responses, ${ }^{18,19}$ is a major way to induce the production of AMP genes in enterocytes, whereas conventional $\mathrm{NF}-\kappa \mathrm{B}$ signaling appears to be restricted to a few cell types including ISCs and so-called copper cells (CCs). A shared characteristic of these cell types is the strictly localized nature of the response, a mechanism that distinguishes between pathogens and commensals based on their invasiveness rather than on recognition of MAMPs.

\section{RESULTS}

Oral infection induces an epithelial antimicrobial response and activation of dFoxO signaling

In Drosophila, oral infection with a variety of pathogenic bacteria is able to induce an immune response in different parts of the intestinal epithelium. ${ }^{20}$ To precisely identify the regions where this immune response takes place, the expression of AMP-coding genes was monitored using fly strains containing AMP-promotor-GFP (green fluorescent protein) constructs. Flies were orally infected with the Gram-negative bacterium Serratia marcescens (Db11; Supplementary Figure 1 online). Representative results obtained with two different AMP-reporter lines are shown (attacin::GFP and defensin::GFP; Figure 1). The attacin::gfp line showed strong expression in the proventriculus in response to oral infection (Figure 1b), whereas the matching controls show much weaker basal signals (Figure 1a). In the defensin::gfp reporter line, mainly the regions of the anterior midgut were labeled in a mosaic-like manner (Figure 1c and d). Moreover, we orally infected a nuclear thor-lacZ (4E-BP-lacZ) line ${ }^{21}$ with Serratia DB11 and could visualize strong infection-induced expression in enterocytes of the intestine (E, control; F, infected 
for $6 \mathrm{~h})$. As thor $(4 E-B P)$ is a canonical FoxO target gene, this induced expression indicates that infection activates FoxO signaling in enterocytes of the intestine. To evaluate the relevance of $\mathrm{dFoxO}$ for antimicrobial responses in the intestines, transcript levels of selected immune-relevant genes were analyzed in $w^{1118}$ and foxo $0^{21 / 21}$ animals either in response to infection $(6 \mathrm{~h})$ or were left undisturbed $(\mathrm{G})$. The AMP genes diptericin, attacin $B$, and attacin $A$ were significantly upregulated in intestines of wild-type animals in response to an infection, similar as the pattern recognition molecule pgrp-sc, whereas the increase in transcript levels observed for upd3 was not statistically significant. Foxo ${ }^{21 / 21}$ animals on the other hand showed no significant increase in transcript levels of any of these genes in response to oral infection, indicating that $\mathrm{dFoxO}$ is required to launch this epithelial antimicrobial response (Figure 1g).

To further evaluate the signaling mechanisms that underlie the infection-induced expression of AMP genes in the intestinal epithelium, we performed in situ analyses of the major pathways involved in epithelial immunity of the fly. These pathways comprise $\mathrm{Ca}^{2+}$-mediated activation of ROS production by Duox and NF- $\kappa \mathrm{B}$ - as well as FoxO-mediated activation of AMP production. To visualize pathway activation, suitable enhanced fluorescent proteins-tagged proteins that show translocation to other cellular compartments in response to this pathway activation were used. This was achieved by targeting expression of the corresponding fusion constructs (using UAS-PLC- $\beta$-RFP, UAS-Relish-GFP, or UAS-dFoxoGFP) to intestinal enterocytes (ECs; using the NP1-Gal4 driver line). Oral infection with $S$. marcescens induced translocation of PLC- $\beta$ from the cytoplasm to the plasma membrane, which indicated that the $\mathrm{Ca}^{2+}$-PLC- $\beta$-Duox pathway responds to infection under these conditions (Figure 2a), a reaction that has already been reported as a response to intestinal infection. ${ }^{13}$ In addition, infection ( $S$. marcescens, OD25 for $24 \mathrm{~h}$ ) was able to activate the CaLexA system in ECs that effectively monitors prolonged $\mathrm{Ca}^{2+}$ signaling in target cells. ${ }^{22}$ Moreover, infection triggered nuclear translocation of FoxO in ECs of the fly's intestine, with an almost complete nuclear translocation was observed $24 \mathrm{~h}$ post infection (Figure $2 \mathrm{c}$ ). In contrast, we did not observe any nuclear translocation of Relish, the NF- $\kappa \mathrm{B}$ factor of the IMD pathway, in response to infection in ECs, indicating that the corresponding IMD pathway is not activated in these cells (Figure 2b). A dose-response study using different doses of infectious bacteria revealed that FoxO translocation in ECs is induced at low pathogen concentrations (OD1), whereas that of Relish is not induced at all. Surprisingly, Dorsal, an NF- $\kappa \mathrm{B}$ factor that has been associated with Toll signaling, also shows nuclear translocation in response to oral infection (Supplementary Figure 2). In addition, time series experiments revealed that FoxO translocation into the nucleus of ECs was $8 \mathrm{~h}$ post infection at an advanced stage. In these experiments, we employed conventional immunohistochemistry to show that the use of tagged reporter proteins is a suitable approach and gives very similar results (Supplementary Figure 3).

\section{AMP synthesis in ECs can be activated by local FoxO signaling}

To achieve spatial and temporal control of expression, we employed the TARGET system, ${ }^{23}$ which adds a temperaturesensitive Gal4 inhibitor to the Gal4/UAS system (NP1-GAL4; tubGal80 ${ }^{t s}$ ). By shifting the temperature from the restrictive temperature $\left(19^{\circ} \mathrm{C}, \quad \mathrm{Gal} 80\right.$ is active, no Gal4-induced expression) to the permissive temperature $\left(30^{\circ} \mathrm{C}, \mathrm{Gal} 80\right.$ is inactivated, Gal4-dependent transcription is possible), we can switch on either the IMD-, or the Toll pathway as well as FoxO-dependent responses in ECs only. Subsequently, we quantified the transcript levels of five major AMP genes (attacin, drosomycin, diptericin, defensin, and drosocin) in material derived from manually isolated intestines using quantitative real-time PCR (qRT-PCR). Intestines were isolated from the experimental animals $24 \mathrm{~h}$ post induction. Animals of the same genotype that were kept at the restrictive temperature served as controls. In (Figure 3a), we ectopically activated FoxO-dependent signaling by simple overexpression of dfoxo (NP1-GAL4; tubGal80 ${ }^{\text {ts }}$ X UAS-dfoxo). Except drosocin, all other AMP genes showed a strong and significant increased expression, which was for attacin and diptericin several hundred-fold. Moreover, a group of genes that was shown above (Figure 1g) to be inducible by oral infection can also be induced by ectopic foxo overexpression (Supplementary Figure 4). As infection-induced expression of these genes (comprising attA, attB, PGRP-SC, thor, or Lip4) depended on functional $\mathrm{dFoxO}$, a central role of $\mathrm{dFoxO}$ dependent signaling for intestinal antimicrobial responses is further supported. Ectopic expression and release of PGRP-LE by ECs (NP1-GAL4; tubGal80 ${ }^{\text {ts }}$ X UAS-pgrp-le; Figure 3b), which activates the IMD pathway in all cells contain the entire IMD-pathway machinery, especially the central PPR PGRP-LC, led to only minor increases in transcript levels. Only attacin showed a slightly increased expression, which implies that the majority of cells in the intestine does not express a fully functional IMD pathway. If we overexpress the major PPR PGRP-LC on the other hand (NP1-GAL4; tubGal80 ${ }^{\text {ts }}$ X UASpgrp-lc), massively increased transcript levels for all AMP genes were observed. For attacin, diptericin, and drosocin the transcript levels increased several hundred fold (Figure 3c). This implies that the IMD-pathway machinery is functional in ECs; however, the major pattern recognition molecule PGRP-LC is not present at all or only in very low concentration. Chronic and prolonged overexpression of pgrp-lc in ECs only led to premature death of all tested animals. To activate Toll signaling in the intestine, we ectopically expressed a constitutively active version of spaetzle in ECs, which is the ligand of the Toll pathway (NP1-GAL4; tubGal $80^{\text {ts }} \mathrm{X}$ UAS-sp $z^{C A}$; Figure 3d). In this case, only very slightly increased expression of some AMP genes could be observed.

To learn whether the effects of FoxO activation on the induced transcription of AMP genes is direct or indirect, we performed an in silico approach to identify potential FoxO-binding sites in the presumptive promoter regions of 
the genes of interest. This was performed with conventional blast analyses employing between 1.5 and $2 \mathrm{~kb}$ upstream of the transcription start site. Exactly, as described by Becker et al., ${ }^{24}$ we identified conserved FoxO-binding sites (TTGTTTAC) only in the cecropin and drosomycin promoter regions. The general forkhead-binding site on the other hand could be identified in most promoter regions (TNTTTAN). Thus, it is not possible to decide whether the effects are direct (mediated via the conserved FoxO-binding site or the more general forkhead-binding site) or are indirect, mediated via a yet unknown signaling mechanism.

\section{Animals deficient in functional FoxO signaling show a reduced survival and a higher bacterial load in response to oral infection by $S$. marcescens}

To elucidate whether FoxO and/or IMD signaling are relevant for surviving an oral infection, we used lines that are impaired in either the FoxO- $\left(f_{0 x o^{21 / 21}}\right)$ or the IMD pathways $\left(\mathrm{rel}^{\mathrm{E3} 8}\right)$ together with the matching wild-type $\left(w^{1118}\right)$ controls, and infected them with $S$. marcescens. Males and females of all genotypes were tested independently. The experiments showed that animals of both sexes that were impaired in FoxOmediated signaling (foxo ${ }^{21 / 21}$ homozygotes) or Relish ( $\mathrm{rel}^{\mathrm{E} 38}$ ), the NF- $\kappa \mathrm{B}$ factor of the IMD pathway, show reduced survival
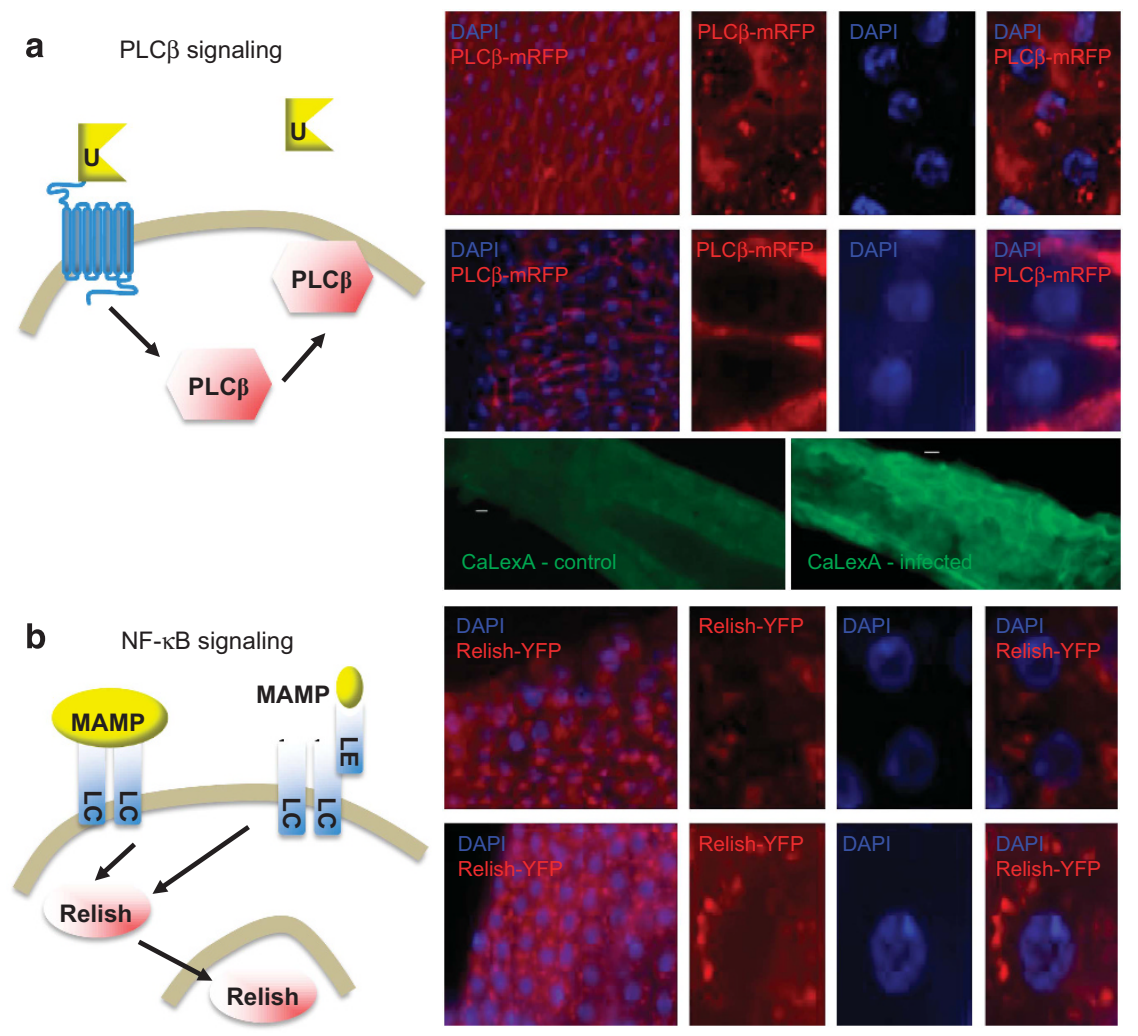

C

dFoxO signaling
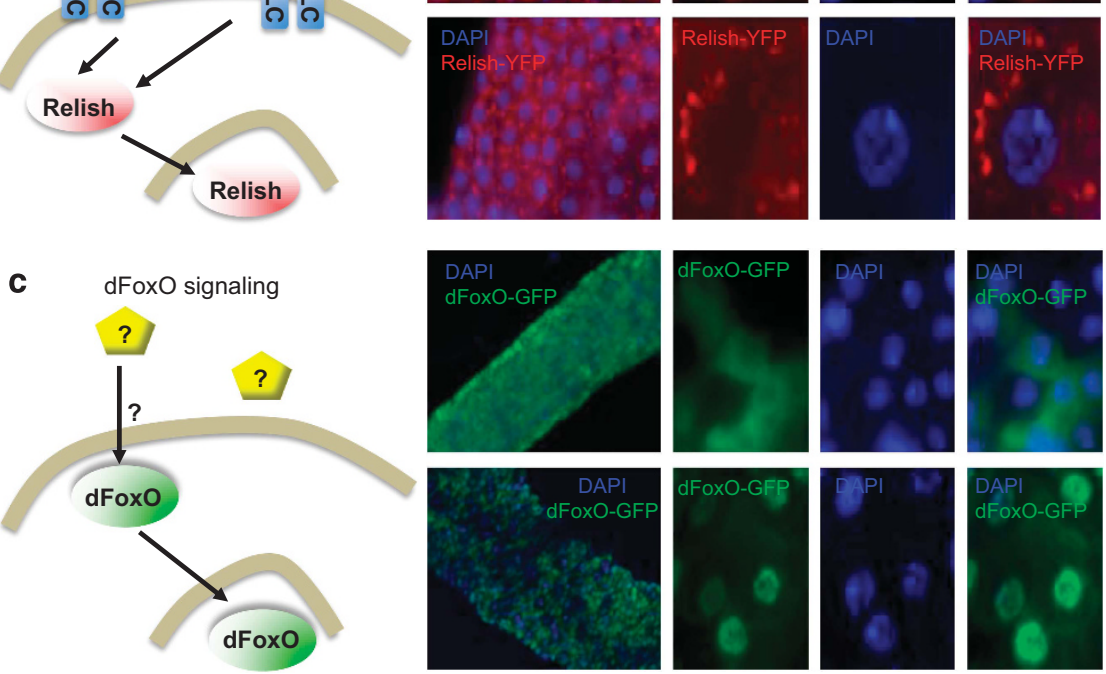

Figure 2 Oral infection with Serratia marcescens Db11 activates different immune-dependent signaling pathways in enterocytes. Different reporter constructs targeted to enterocytes (ECs) were used to elucidate the signaling pathways involved in the epithelial immune response to an oral infection with S. marcescens Db11. NP1-Gal4 > UAS-PLCB-mRFP (a), which express a RFP-tagged fusion with PLCß in ECs only, showed cytoplasmic localization under control conditions (top row), whereas infection-induced translocation to the membrane (bottom row). Infection was able to activate green fluorescent protein (GFP) expression in CaLexA flies, indicative for prolonged $\mathrm{Ca}^{2+}$ activation in enterocytes. NP1-Gal4> UAS-Relish-YFP drives expression of a YFP-tagged Relish protein to ECs (b). Nuclear translocation was not observed under control conditions (top row), or following infection with S. marcescens (bottom row). NP1-Gal4 > UAS-dFoxO-GFP targets a GFP-tagged dFoxO protein to ECs (c). Under control conditions (top row), the signal was found in the cytoplasm, while infection with $S$. marcescens induced its nuclear translocation (bottom row). Bar $=20 \mu \mathrm{m}$. 

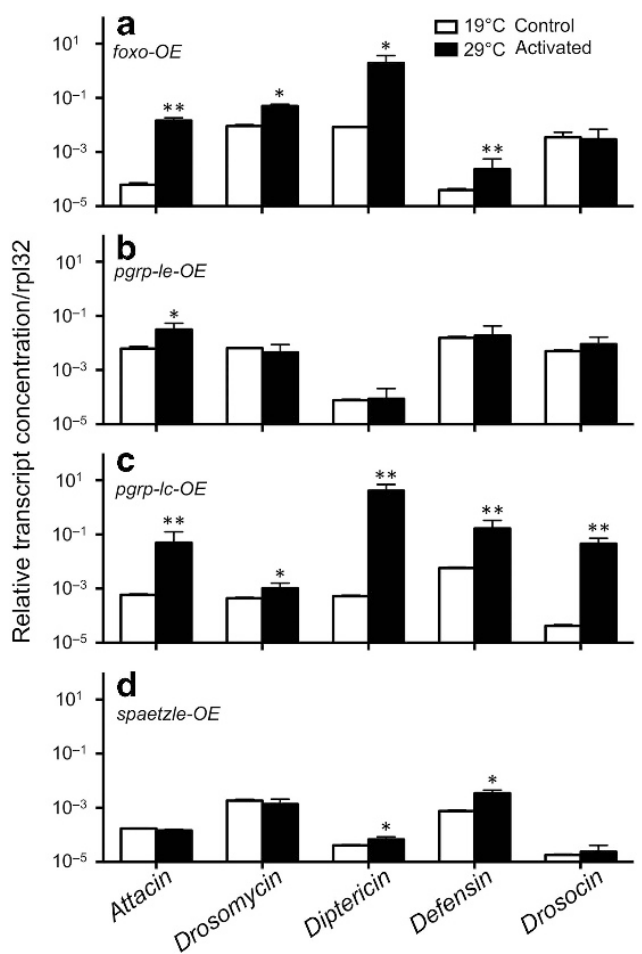

Figure 3 Effects of ectopic activation of intestinal NF- $\mathrm{kB}$ - or FoxOdependent signaling on the expression of antimicrobial peptide genes. Using an inducible expression system (TARGET system) targeted to the intestine, NF-kB- or FoxO-dependent signaling was induced in the intestine, and the effect of this intervention on the expression of selected antimicrobial peptide genes was quantified. Ectopic expression of the different signaling pathways was induced by shifting animals from the restrictive $\left(19^{\circ} \mathrm{C}\right.$, white bars) to the permissive temperature $\left(29^{\circ} \mathrm{C}\right.$, black bars) and measuring the transcript levels using quantitative real-time PCR (qRT-PCR) with material from control animals $\left(19^{\circ} \mathrm{C}\right)$ and induced ones $\left(29^{\circ} \mathrm{C}\right)$. Ectopic activation of FoxO signaling was achieved by overexpression of FoxO (a); PGRP-LE was used to activate the IMD pathway in all completely immune-competent cells (containing PGRP-LC (b)); and PGRP-LC was used to activate the IMD pathway independent on the presence of this pattern recognition receptor (c). In d, a constitutively active spaetzle (spz) was used to induce Toll signaling in the intestine (d). Shown are the mean values of three to four biological replicates \pm s.e.m. ${ }^{\star} P<0.05,{ }^{\star \star} P<0.001, t$-test.

rates in response to oral infection with $S$. marcescens compared with control animals (Figure $\mathbf{4 a - d}$ ). In order to evaluate whether the corresponding genotypes ( $f o x o^{21 / 21}$ and $\mathrm{rel}^{\mathrm{E3} 8}$ ) have indeed reduced resistance to oral infections, we normalized the survival curves for both genotypes $\left(\right.$ foxo $\mathrm{O}^{21 / 21}$ and $\mathrm{rel}^{\mathrm{E3} 8}$ ) according to the median lifespan of their untreated group relative to that of untreated $w^{1118}$. The normalized data of male as well as female foxo ${ }^{21 / 21}$ revealed a statistically significantly reduced lifespan compared with infected $w^{1118}(P<0.0001)$. The reduced lifespan observed in infected male as well as female $\mathrm{rel}^{\mathrm{E38}}$ flies in comparison with infected $w^{1118}$ was not statistically significant (males $P=0.245$, females $P=0.35$ ).

Moreover, we developed a novel type of bacterial load assay and performed it with the three genotypes mentioned above $\left(\mathrm{foxo}^{21 / 21}, \mathrm{rel}^{\mathrm{E38}}\right.$, and $\left.w^{1118}\right)$, both in males and in females (Figure 4e,f). In order to allow quantification of the number of
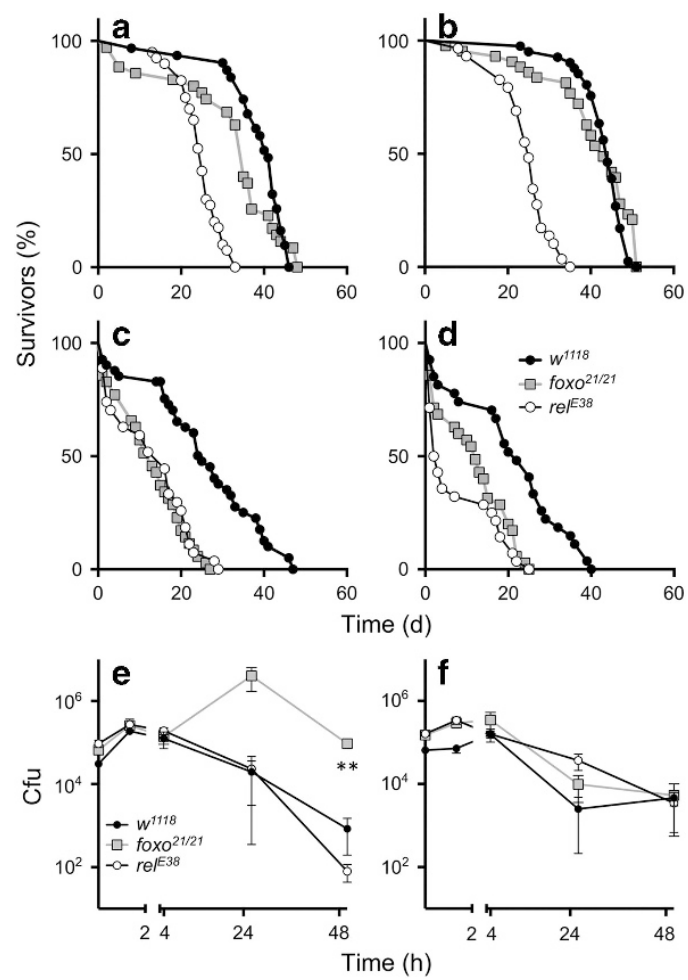

Figure 4 Effect of oral infection on lifespan and bacterial load in IMD-and $\mathrm{dFoxO}$-deficient animals. Males (a and $\mathbf{c}$ ) and females (b and $\mathbf{d}$ ) of the different genotypes $w^{1118}$ (control, black), re ${ }^{38}$ (white), foxo ${ }^{21 / 21}$ (grey) were fed with $D b 11\left(\mathrm{OD}_{600}>50 ; \mathbf{c}\right.$ and d) or diluent only ( $5 \%$ sucrose; $\mathbf{a}$ and b) throughout their adult life, and the number of survivors was quantified every second day. $n=100$ individuals each. Statistical analysis was performed with the Mantel-Cox log-rank test (Supplementary Table 2). For the bacterial load assays, adult animals of males (e) and females (f) were fed with Serratia DB11 (OD1 in $5 \%$ sucrose) for $2 \mathrm{~h}$ that was supplemented with blue food dye to allow quantification of the ingested bacteria (starting points) and then placed on normal medium. Labeling of genotypes was as in $\mathbf{a}-\mathbf{d}$ and the mean values \pm s.e.m. (four biological replicates) are displayed. ${ }^{* *} P<0.01, t$-test.

ingested bacteria, flies were fed with a solution containing $S$. marcescens (OD1) in $5 \%$ glucose that was supplemented with a blue food dye for only $2 \mathrm{~h}$. Photometric determination of the amount of ingested, colored liquid allowed quantification of the total number of ingested bacteria. This assay showed that, although control animals and animals impaired in FoxO or IMD signaling ingested the same number of bacteria, a substantially (statistically significant) higher amount of Serratia was seen in $f \circ x o^{21 / 21}$ males at $48 \mathrm{~h}$ post infection (Figure 4e), whereas this was not seen in females or either in $\mathrm{rel}^{\mathrm{E3} 8}$ males or females (Figure $4 \mathbf{e}$ and $\mathbf{f}$ ). These results imply that especially foxo $0^{21 / 21}$ males have problems in controling the number of ingested bacteria in the intestine.

\section{Characterization of intestinal cells that launch an immune response following IMD activation}

Despite the observed insensitivity of the ECs' IMD pathway to oral infection, an important role of this signaling pathway for intestinal immunity has been documented. ${ }^{25}$ To elucidate whether other cells of the intestine are able to trigger an 
IMD-dependent immune response, and to identify these reactive cell populations, we adopted an overexpression approach that allowed us to identify the complete set of IMD-responsive cells in the intestine. Organ-wide release of the soluble PPR PGRP-LE into the intestinal lumen activates all cells expressing PGRP-LC, the major PRR of the IMD pathway. Furthermore, as drosomycin is a target gene of the IMD pathway in epithelial tissues, its promoter activity can be used as a measure of pathway activity. By crossing the EC-specific driver line NP1-GAL4 with UAS-FLAG-pgrp-le; Drs-GFP we could simultaneously induce enhanced release of PGRP-LE and measure cell responsiveness by GFP reporter activity. To optimize the detection of GFP signals, the reporter was detected using immunohistochemistry. We could not detect any signal in ECs; however, three different cell regions/populations in the midgut of Drosophila showed a very strong and reproducible signal. These regions comprise the proventriculus (Figure 5a-c), a large group of small and dispersed cells, as well as the anterior region of the midgut (Figure 5d). In addition to the proventriculus, whose activation during gastrointestinal tract infection has been characterized in great detail, the other cell populations have also been identified using double-labeling experiments. The second cell group that shows expression of AMP in response to IMD-pathway activation comprises small cells scattered over the entire midgut. Structurally, these cells resemble ISCs. Colocalization experiments using GFP (Figure $\mathbf{5 e}, \mathbf{h}, \mathbf{g}$ and $\mathbf{j}$, green channel) to detect drosomycin-promotor activity, and the stem cell-marker Delta (Figure $\mathbf{4 f}, \mathbf{i}, \mathbf{g}$ and $\mathbf{j}$, red channel), showed that these drosomycin-expressing cells are indeed ISCs (Figure 5d-j).

The third group of cells able to launch an IMD-dependent immune response shows characteristics of so-called CCs. These cells constitute the acidic regions of the anterior midgut and are very large, cup-shaped cells with a deep invagination connected to the intestinal lumen via a small pore. As CCs are metal cation-incorporating cells, they accumulate metal cations to very high levels. ${ }^{26}$ Feeding larvae with $\mathrm{CuSO}_{4}$-containing medium for $24 \mathrm{~h}$ allowed their identification because they showed a marked ultraviolet-induced fluorescence (Figure 5k and $\mathbf{~}$ ). We performed time course experiments of Relish- and Foxo translocation in CCs. Only Relish translocated into the nucleus in response to an infection, whereas Foxo did not (Supplementary Figure 5). The cellular organization of CCs (Figure $\mathbf{5 m}$ ), which is hidden from the central lumen of the intestine while launching an immune response, resembles Paneth cells in the crypts of the small intestine. Paneth cells, the major immune-competent cells in the human intestine, express a characteristic set of genes, including those for AMP, lysozymes, metallothioneins, and soluble phospholipases A2 (sPLA2s). ${ }^{27}$ Thus, we tested whether homologous genes are expressed in the CCs of the anterior midgut. The proventriculus, a part of the anterior midgut between the proventriculus and the CC region, and the CC region itself, were isolated manually. qRT-PCR with complementary DNA derived from the different regions revealed that in the CC region major genes that had been used to characterize Paneth cells were present (Figure 5n). In the other adjacent midgut regions, this was not the case. Notably, metallothionein and sPLA2 were absent or were present at only very low levels (Figure 5n).

\section{DISCUSSION}

The intestinal immune system of the fruit fly is made of three almost independently operating modules, allowing the launch of an appropriate immune response against infection while protecting the indigenous microbiota. Keeping the response local appears to be strategy of this multilayered immune system. In the current study, we showed that dFoxO-mediated signaling in the intestine is necessary to survive oral infection by $S$. marcescens and to control the intestinal bacterial load. Moreover, induction of epithelial antimicrobial responses also depends on functional dFoxO signaling in ECs of the intestine. In contrast, functional IMD signaling is restricted to very few cell types of the intestine that have very peculiar structures (the proventriculus, the CCs, and the ISCs).

The architecture of the intestinal immune system has to follow simple rules in order to maintain tolerance against the microbiota while retaining the capacity to fight pathogens effectively. Most importantly, immune responses have to be kept local and the dissemination of antimicrobial compounds has to be restricted. In the mammalian intestine, AMPs are produced into the mucosal layer, forming a gradient from the cell membrane to the apical part of the mucus. Although bacteria attaching to the mucus layer come into contact with antimicrobial compounds, the deeper they invade into the layer toward the ECs, the higher the AMP concentration is. ${ }^{28}$ Consequently, while being protected against invading pathogenic bacteria, the indigenous microbiota is not affected. ${ }^{29}$ The peritrophic membrane in insects may be the functional equivalent to the mucus layer. It separates the intestinal lumen with the bacteria from the epithelial cells, ${ }^{30}$ and has an important role in pathogen resistance in Drosophila. ${ }^{31}$ Furthermore, the proventriculus, which shows production of AMPs following oral infection, also produces the peritrophic membrane, indicating that this region of the intestine plays a critical role for the protection against pathogenic bacteria.

Our work has shown that, depending on the intestinal cell type, the production and release of AMPs is controlled by at least two different mechanisms. Activation of the IMD pathway, which is the sole innate immune pathway operative in epithelia, depends on the recognition of bacterial MAMPs by major PRRs such as PGRP-LC. Our finding that dorsal translocates in ECs in response to an infection is currently not understood at all. Dorsal is devoted to the Toll pathway that is believed to be nonoperative in epithelia of the fly. ${ }^{32,33}$ Our attempts to activate Toll signaling in the intestine that failed to induce a pronounced antimicrobial response supported these earlier studies. Thus, a functional role of dorsal in intestinal immunity remains to be elucidated. A major cell population of the intestine, the ECs, is always at risk of being activated by MAMPs derived not only from pathogens, but also from the 

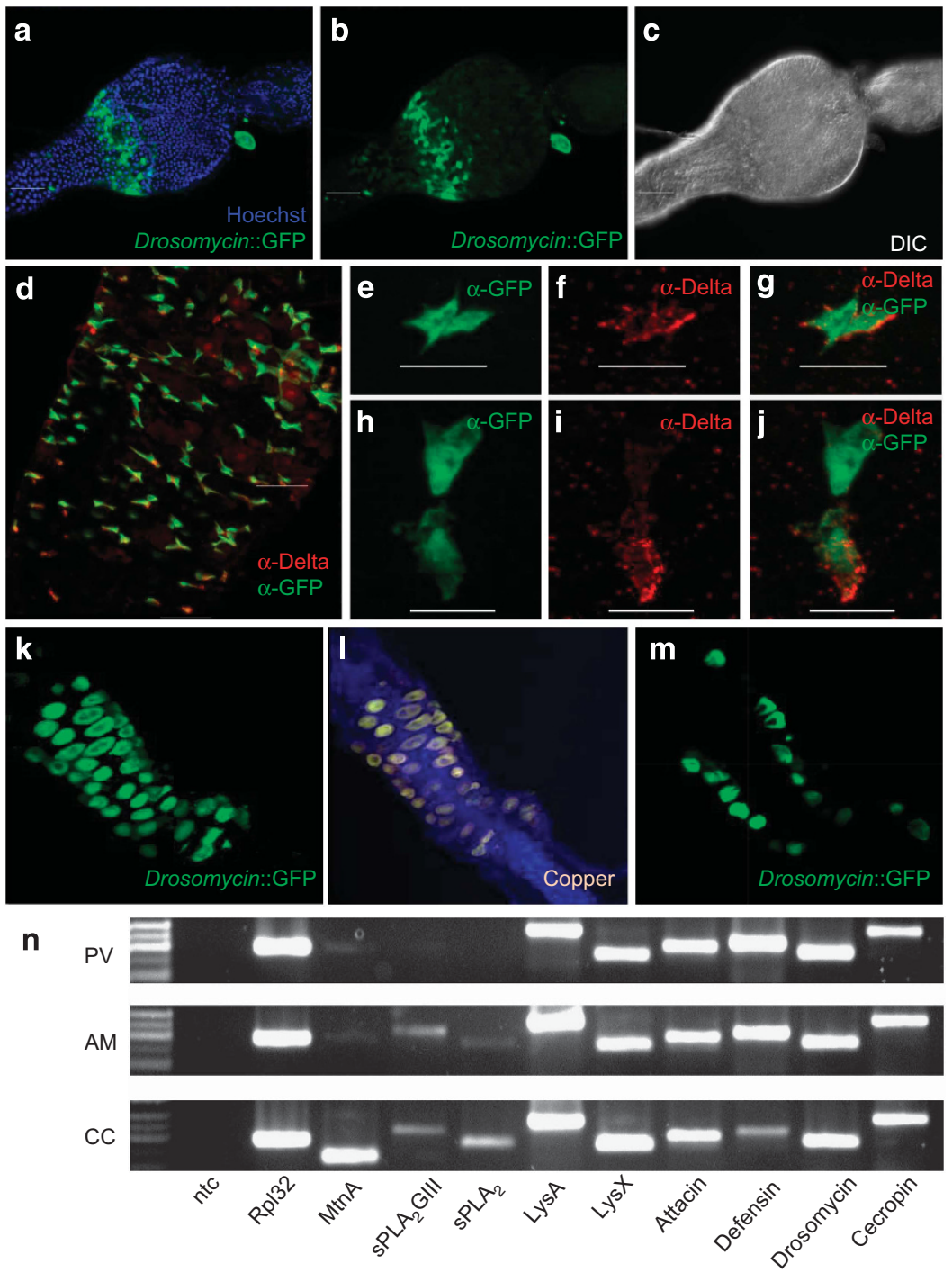

Figure 5 IMD triggers a regionalized AMP response in the adult midgut. Ectopic activation of the IMD pathway was achieved by overexpression of the soluble pattern recognition receptor (PRR) PGRP-LE in enterocytes (ECs) (NP1-Gal4 $\times$ UAS-FLAG-pgrp-le). Activation of the IMD pathway is shown by the occurrence of green fluorescent protein $(G F P)$ under the control of a drosopmycin promotor. Drosomycin-promotor activity was observed in the proventriculus (a-c) in only a specific region. An overlay of 4,6-diamidino-2-phenylindole and green fluorescent protein (GFP) staining (a), GFP-staining only (b), and transmission light of the structure (c) are shown. Throughout, most regions of the midgut stem cells were labeled (d-j). Anti-GFP (e and $\mathbf{h}$ ), anti-delta staining ( $\mathbf{f}$ and $\mathbf{i})$, or overlays of both signals ( $\mathbf{d}, \mathbf{g}$ and $\mathbf{j})$ are shown. In the copper cell (CC) region, characteristic cells were labeled ( $\mathbf{k}$ and $\mathbf{m}$ ). Feeding 1-mM CuSO${ }_{4}$-treated medium induced a fluorescent signal in the same cells (I). Confocal analysis revealed the cup-shaped structure of these cells, an opening with only a small pore toward the intestinal lumen (m). (n) Real-time PCR (RT-PCR) analysis performed with different regions of the larval anterior midgut, the proventiculus (PV), anterior midgut (AM), and the CC. Shown are the results of the RT-PCR experiments performed with oligonucleotides specific for the following gene products: LysA = lysozyme A, LysX=lysozyme X, attacin, defensin, drosomycin, cecropin, metallothionein $A=(M \operatorname{tnA})$, soluble phospholipase $A 2$ genes ( $\left(\mathrm{PLA}_{2}\right.$ and $\mathrm{sPLA} \mathrm{A}_{2} \mathrm{G}$ III), as well as controls (rpL32) and the no template control (ntc).

indigenous microbiota. The architecture of major parts of the fly's epithelial immune system presumably aims to reduce impairments of the microbial community in case of an immune response. This is achieved by restricting responses to the site of direct pathogen contact. In the Drosophila intestine, the production of ROS by ECs appears to be one of the first responses against invading pathogens. ${ }^{34-37}$ Duox-dependent production of these toxic compounds is triggered locally by bacterial uracil and remains localized because ROS diffusion is spatially limited. ${ }^{38}$ It has been proposed that this induced production of ROS is the most important part of the intestinal defense system. ${ }^{39}$ The synthesis of AMP complements the production and release of ROS, therefore, creating a multilayered intestinal immune response. ${ }^{39}$ To ensure locality of the second type of antimicrobial responses in ECs, the production and release of AMPs we identified are not dependent on MAMP recognition via conventional innate immune signaling-pathway activation. This view is supported by our overexpression experiments, where ectopic expression of PGRP-LE was not able to induce substantial AMP expression in the intestine, 
while that of PGRP-LC was. Released PGRP-LE can only activate the IMD pathway in those cells that express the major PPR PGRP-LC at a certain concentration. Therefore, it is ideally suited to identify and characterize those cells that are fully immune (meaning IMD)-competent. In the intestine, the majority of ECs is obviously not. The experiments, where PGRP-LC was ectopically overexpressed, clearly showed that ECs are able to express AMPs in an IMD-dependent manner, but that these cells express very low levels or even no PGRP-LC under normal conditions, thus shutting down conventional MAMP-based activation of innate epithelial immune responses in these cells. Our observation that prolonged and strong IMD activation (via ectopic PGRP-LC expression) in ECs led to premature death of the animals further supported our hypothesis that IMD signaling has to be restricted in enterocytes. We propose that the expression of AMP genes entirely depends on danger-mediated signaling acting locally at the site of damage. This response is presumably FoxO-dependent and ensures that focal rather than massive release of AMPs is the result of a pathogen-epithelium interaction. Our results, especially the absence of induction of AMPs in response to infection in FoxO-impaired animals, support this hypothesis. Avoiding conventional innate immune signaling therefore would allow differentiation between indigenous microbiota and pathogenic (invasive) bacteria. Only pathogenic bacteria try to invade, coming into contact with the epithelium, and thus only these regions are endangered and need protection. FoxO-dependent local release of AMPs is therefore the ideal way to react to these pathogenic bacteria. Another aspect of FoxO-mediated signaling in the intestine complements the antimicrobial epithelial response. Following oral infection with E. carotovora, activation of the FoxO/JNK axis induces an antioxidative response, ${ }^{40}$ which may protect epithelia from damage caused by Duox-generated ROS during an antimicrobial response, thus ideally complementing the multifaceted intestinal response to pathogens. An alternative role of FoxO has very recently been described. In ageing flies, immune senescence of the gut is associated with increased FoxO activity in ECs. This increased FoxO activation disturbs both, the $\operatorname{lipid}^{41}$ as well as the immune homeostasis of the intestine. $^{42}$ The latter response is caused by a FoxO-mediated reduction PGRP-SC expression, which is a potent inhibitor of NF-KB-signaling pathways in the fly's intestine. ${ }^{42}$ Guo et al. proposed that the positive effect of FoxO activation is indirect, mediated via releasing inhibition of the IMD-signaling pathway. In contrast, our data are perfectly congruent with the assumption that FoxO activation directly induces an epithelial immune response. Nevertheless, it is possible that FoxO directly interacts with IMD signaling within the EC, thus utilizing parts of the innate immune machinery of these cells for a stress/danger-induced activation of AMP gene expression.

Despite the role of FoxO-dependent production of AMPs in ECs, IMD-dependent production of AMPs is of great importance for intestinal immunity. We found it in specific places within the intestine, presumably to protect these cells. In addition to the proventriculus that forms the peritrophic membrane, two other cell types, the CCs and ISCs, also show IMD-dependent production of AMPs. CCs are secreting cells that are responsible for acidification and detoxification. ${ }^{26}$ In contrast to other intestinal cells, their major surface area is hidden from the intestinal lumen. They form a structure comparable to an intestinal crypt in a single cell, connected to the intestinal lumen only through a small pore. Pathogenic bacteria trapped in this invagination represent a very severe risk. When bacteria enter these invaginations they are no longer transported together with the intestinal content, which represents an ideal situation for local colonization. Upon invasive S. marcescens infections, these bacteria have been identified in the CCs' invaginations ${ }^{43}$ and, therefore, these invaginations need very effective antimicrobial systems. As shown recently, CCs are AMP-producing cells activated through monomeric DAP-type peptidoglycan. ${ }^{44}$ Besides having similar architecture, Paneth cells and CCs of the fly share additional commonalities regarding their role as immune cells guarding these structures, as well as their expression patterns.

ISCs are the last group of cells involved in IMD signaling. Presently, little is known about the protective role of ISCs; in particular, it is unclear whether they have a role in AMP production and why such a peptide synthesis is detectable in these cells at all. Taking their small size into account, the production of AMPs by ISCs should not have any systemic effect or any effect on bacteria residing in the intestinal lumen. Production of AMPs by ISCs has recently been described. Michael Boutros group was able to show that MAPK-mediated inhibition of NF- $\mathrm{KB}$ signaling induces AMP production in these cells, which presumably aids the survival of ISCs, even if pathogens managed to invade the intestinal epithelium. ${ }^{45}$ This situation again resembles that of Paneth cells that guard and protect adjacently located ISCs. Drosophila ISCs perform the role of both mammalian Paneth cells and ISCs.

Taken together, our experiments showed that the intestinal immune system is made of multiple modules leading to either local ROS production or local AMP release. This architecture ensures strictly localized antimicrobial responses, enabling efficient pathogen removal while protecting the indigenous microbiota. FoxO-dependent signaling has a central role in defense against pathogenic bacteria.

\section{METHODS}

Fly strains and maintenance. The following strains were used in this study: NP1-GAL4 and NP1-GAL4; tubGal80 ${ }^{\text {ts }} 46$ UAS-PGRP-LC, UAS-PGRP-LE; ${ }^{47}$ UAS-FOXO::GFP; ${ }^{48}$ UAS-Relish::YFP, ${ }^{49}$ UAS-FOXO,${ }^{50}$ Drs-GFP, ${ }^{51}$ dfoxo, ${ }^{25}$ and dfoxo, ${ }^{21,52} \mathrm{Rel}^{\mathrm{E} 38}, \mathrm{w}^{1118} \mathrm{e}[\mathrm{s}]$, thor-lacZ, UAS$\mathrm{spz}^{\mathrm{CA}}$. Rel ${ }^{\mathrm{E} 38}$, thor-lacZ, and $\mathrm{w}^{1118} \mathrm{e}[\mathrm{s}]$ were obtained from the Bloomington Stock Center. The CaLexA flies (LexAop-CD8-GFP-2A-CD8GFP/LexAop-CD8-GFP-2A-CD8-GFP; UAS-mLexA-VP-NFAT C del(42), LexAop-CD2-GFP/TM6B) were generously provided by Ronald Kühnlein (Göttingen, Germany). Flies were kept at $25^{\circ} \mathrm{C}$ on standard Drosophila medium (cornmeal, yeast, glucose, sugar beet molasses, agar, and preservatives). After hatching they were kept on normal medium for 7 days. Mated females were used for experiments.

Oral infection. Overnight bacterial cultures (S. marcescens (Db11)) were grown at $30^{\circ} \mathrm{C}$ in lysogenic broth medium supplemented with 
Streptomycin $\left(10 \mu \mathrm{g} \mathrm{ml}^{-1}\right)$. Concentrated cultures were resuspended in $5 \%$ sucrose. For infection, flies were moved to vials covered with a filter disk saturated with $300 \mu$ of the bacterial suspension $\left(\mathrm{OD}_{600} 50\right)$. Controls were fed the same way, except that filter disks were saturated with $5 \%$ sucrose only. The flies were then kept at $30^{\circ} \mathrm{C}$.

Bacterial load assay. To assess the ability of different Drosophila strains to cope with ingested pathogenic bacteria, we adapted conventional bacterial load assays to allow quantification of the number of ingested pathogenic bacteria. Adult flies (4-6 days after hatching) were starved for $2 \mathrm{~h}$, followed by addition of $S$. marcescens (OD 1 final concentration) resuspended in $5 \%$ glucose that was supplemented with food dye $(0.5 \%$ blue dye E133 brilliant blue, Roth, Karlsruhe, Germany). Animals were allowed to feed for $2 \mathrm{~h}$, and only those animals with visual blue coloration of the abdomen were used for the bacterial load assay. In order to quantify the amount of ingested bacteria, groups of three animals (males and females separated) were homogenized and their $\mathrm{OD}_{600}$ was measured spectrophotometrically. A standard curve obtained by measuring dilutions of the colored inoculum was used to quantify the initial bacterial load. After defined time points, groups of three animals each were taken, homogenized, and plated onto lysogenic broth agar plates (Streptomycin $10 \mu \mathrm{g} \mathrm{ml}^{-1}$ ). After an incubation at $30^{\circ} \mathrm{C}$ overnight, colony-forming units were counted. Each point is the mean of five biological replicates (mean values \pm s.e.m.).

Immunohistochemistry CaLexA system and image acquisition. Immunohistochemistry was performed as previously described. ${ }^{53}$ Translocation of transcription factors (Relish and FoxO) and of PLC $\beta$ was assessed using enhanced fluorescent proteins-tagged versions of the corresponding proteins targeted to the intestine using the NP1Gal4 driver. Using the same NP1-Gal4 driver line, CaLexA-ready flies were produced that allowed monitoring its activation in ECs.

LacZ staining. After 5 days, thor-lacZ female flies were starved for $16 \mathrm{~h}$ overnight at $25^{\circ} \mathrm{C}$ on an agar-containing medium with watersaturated filter paper to avoid dehydration. For infection, a fresh overnight culture of $S$. marcescens (see above) was used. A bacterial suspension of $\mathrm{OD}_{600} 10$ was fed for $6 \mathrm{~h}$. Controls were fed the same with $5 \%$ sucrose only. Subsequently, the intestines were dissected in $1 \times$ phosphate-buffered saline and then were fixed in $0.75 \%$ glutaraldehyde for $15 \mathrm{~min}$. Tissue was washed three times in fresh $1 \times$ phosphatebuffered saline and incubated in pre-warmed $\left(37^{\circ} \mathrm{C}\right)$ staining solution containing $0.025 \% \mathrm{X}-\mathrm{Gal}$. The samples were incubated for $30 \mathrm{~min}$ at $37^{\circ} \mathrm{C}$. Pictures were immediately taken using the SZX12 microscope (Olympus, Hamburg, Germany) under identical conditions for both types of samples.

qRT-PCR. To evaluate expression levels of AMP-coding genes, we crossed temperature-inducible EC-specific driver lines (NP1-Gal4; tub-Gal $80^{\text {ts }}$ ) with the responder lines UAS-PGRP-LE, UAS-PGRP-LC, UAS-spz ${ }^{\mathrm{CA}}$, or UAS-FoxO. Usually, animals were held at the restrictive temperature $\left(19^{\circ} \mathrm{C}\right)$. Ectopic expression was induced by a brief shift to the permissive temperature $\left(29^{\circ} \mathrm{C}\right)$. Animals of the same genotype, which were not subjected to the inducing temperature shift, served as controls. mRNA isolation, complementary DNA synthesis, and qRT-PCR were performed as previously described. ${ }^{54}$ The amount of the detectable mRNA was normalized to the reference gene RpL32 and relative quantification was performed using the modified ${ }^{\Delta \Delta} C_{t}$ method. $^{33,55}$ For infection experiments, animals of the indicated genotypes were used and the material (isolated intestines) were treated as described above.

Infection experiments. Directly after hatching, flies were separated and males and females were analyzed separately. Oral infection was performed every second day with a fresh solution of Db11 in 5\% sucrose $\left(\mathrm{OD}_{600}>50\right)$. Flies were kept in a bench top incubator at $30^{\circ} \mathrm{C}$ with a $12 \mathrm{~h} / 12 \mathrm{~h}$ light-dark cycle. Results are presented as the percentage of living flies after the first day of infection. Statistical analysis was performed using GraphPad Prism 6.00 (GraphPad Software, La Jolla, CA) with the log-rank (Mantel-Cox) test.

SUPPLEMENTARY MATERIAL is linked to the online version of the paper at http://www.nature.com/mi

\section{ACKNOWLEDGMENTS}

This work was supported by the German research association (DFG) as part of the Cluster of Excellence Inflammation@Interfaces (Project: FoxO factors as key regulators of epithelial homeostasis and the host-microbiome crosstalk) and the CRC1182 (C2). We thank Britta Laubenstein and Christiane Sandberg for excellent technical support. We would also like to thank Kathryn Anderson, Dominique Ferrandon, Tony Ip, Shoichiro Kurata, Bruno Lemaitre, Ronald Kühnlein, and Marc Tatar for flies.

\section{DISCLOSURE}

The authors declared no conflict of interest.

(c) 2016 Society for Mucosal Immunology

\section{REFERENCES}

1. Hooper, L.V., Littman, D.R. \& Macpherson, A.J. Interactions between the microbiota and the immune system. Science 336, 1268-1273 (2012).

2. Cash, H.L., Whitham, C.V., Behrendt, C.L. \& Hooper, L.V. Symbiotic bacteria direct expression of an intestinal bactericidal lectin. Science 313, 1126-1130 (2006).

3. Sato, T. et al. Paneth cells constitute the niche for Lgr5 stem cells in intestinal crypts. Nature 469, 415-418 (2011).

4. Maloy, K.J. \& Powrie, F. Intestinal homeostasis and its breakdown in inflammatory bowel disease. Nature 474, 298-306 (2011).

5. Dupaul-Chicoine, J., Dagenais, M. \& Saleh, M. Crosstalk between the intestinal microbiota and the innate immune system in intestinal homeostasis and inflammatory bowel disease. Inflamm. Bowel Dis. 19, 2227-2237 (2013).

6. Nenci, A. et al. Epithelial NEMO links innate immunity to chronic intestinal inflammation. Nature 446, 557-561 (2007).

7. Fritz, T., Niederreiter, L., Adolph, T., Blumberg, R.S. \& Kaser, A. Crohn's disease: NOD2, autophagy and ER stress converge. Gut 60, 1580-1588 (2011).

8. Franke, A. etal. Genome-wide meta-analysis increases to 71 the number of confirmed Crohn's disease susceptibility loci. Nat. Genet. 42, 1118-1125 (2010).

9. Hugot, J.P. et al. Association of NOD2 leucine-rich repeat variants with susceptibility to Crohn's disease. Nature 411, 599-603 (2001).

10. Leulier, F. \& Royet, J. Maintaining immune homeostasis in fly gut. Nat. Immunol. 10, 936-938 (2009).

11. Dionne, M.S. \& Schneider, D.S. Models of infectious diseases in the fruit fly Drosophila melanogaster. Dis. Models Mech. 1, 43-49 (2008).

12. Ha, E.M. et al. An antioxidant system required for host protection against gut infection in Drosophila. Dev. Cell 8, 125-132 (2005).

13. Ha, E.M. et al. Regulation of DUOX by the Galphaq-phospholipase Cbeta-Ca2 + pathway in Drosophila gut immunity. Dev. Cell 16, 386-397 (2009).

14. Ryu, J.H. et al. An essential complementary role of NF-kappaB pathway to microbicidal oxidants in Drosophila gut immunity. EMBO J. 25, 3693-3701 (2006).

15. Zaidman-Remy, A. et al. The Drosophila amidase PGRP-LB modulates the immune response to bacterial infection. Immunity 24, 463-473 (2006).

16. Paredes, J.C., Welchman, D.P., Poidevin, M. \& Lemaitre, B. Negative regulation by amidase PGRPs shapes the drosophila antibacterial response and protects the fly from innocuous infection. Immunity 35 , 770-779 (2011).

17. Ryu, J.H. et al. Innate immune homeostasis by the homeobox gene caudal and commensal-gut mutualism in Drosophila. Science 319, 777-782 (2008).

18. Lehtinen, M.K. et al. A conserved MST-FOXO signaling pathway mediates oxidative-stress responses and extends life span. Cell 125, 987-1001 (2006). 
19. Zhang, W., Hietakangas, V., Wee, S., Lim, S.C., Gunaratne, J. \& Cohen, SM. ER stress potentiates insulin resistance through PERK-mediated FOXO phosphorylation. Genes Dev. 27, 441-449 (2013).

20. Buchon, N., Broderick, N.A., Poidevin, M., Pradervand, S. \& Lemaitre, B. Drosophila intestinal response to bacterial infection: activation of host defense and stem cell proliferation. Cell Host Microbe 5, 200-211 (2009).

21. Demontis, F. \& Perrimon, N. FOXO/4E-BP signaling in Drosophila muscles regulates organism-wide proteostasis during aging. Cell 143, 813-825 (2010).

22. Masuyama, K., Zhang, Y., Rao, Y. \& Wang, J.W. Mapping neural circuits with activity-dependent nuclear import of a transcription factor. $J$. Neurogenet. 26, 89-102 (2012).

23. McGuire, S.E., Le, P.T., Osborn, A.J., Matsumoto, K. \& Davis, R.L. Spatiotemporal rescue of memory dysfunction in Drosophila. Science 302, 1765-1768 (2003).

24. Becker, T. et al. FOXO-dependent regulation of innate immune homeostasis. Nature 463, 369-373 (2010).

25. Buchon, N., Broderick, N.A., Chakrabarti, S. \& Lemaitre, B. Invasive and indigenous microbiota impact intestinal stem cell activity through multiple pathways in Drosophila. Genes Dev. 23, 2333-2344 (2009).

26. Dubreuil, R.R. Copper cells and stomach acid secretion in the Drosophila midgut. Int. J. Biochem. Cell Biol. 36, 745-752 (2004).

27. Elphick, D.A. \& Mahida, Y.R. Paneth cells: their role in innate immunity and inflammatory disease. Gut 54, 1802-1809 (2005).

28. Meyer-Hoffert, U. et al. Secreted enteric antimicrobial activity localises to the mucus surface layer. Gut 57, 764-771 (2008).

29. Ayabe, T., Satchell, D.P., Wilson, C.L., Parks, W.C., Selsted, M.E. \& Ouellette, A.J. Secretion of microbicidal alpha-defensins by intestinal Paneth cells in response to bacteria. Nat. Immunol. 1, 113-118 (2000).

30. King, D.G. Cellular organization and peritrophic membrane formation in the cardia (proventriculus) of Drosophila melanogaster. J. Morphol. 196, 253282 (1988).

31. Kuraishi, T., Binggeli, O., Opota, O., Buchon, N. \& Lemaitre, B. Genetic evidence for a protective role of the peritrophic matrix against intestinal bacterial infection in Drosophila melanogaster. Proc. Natl Acad. Sci. USA 108, 15966-15971 (2011).

32. Tzou, P. et al. Tissue-specific inducible expression of antimicrobial peptide genes in Drosophila surface epithelia. Immunity 13, 737-748 (2000).

33. Wagner, C., Isermann, K., Fehrenbach, H. \& Roeder, T. Molecular architecture of the fruit fly's airway epithelial immune system. BMC Genomics 9, 446 (2008).

34. Ha, E.M. et al. Regulation of DUOX by the Galphaq-phospholipase CbetaCa2 + pathway in Drosophila gut immunity. Dev. Cell 16, 386-397 (2009).

35. Ha, E.M. et al. Coordination of multiple dual oxidase-regulatory pathways in responses to commensal and infectious microbes in drosophila gut. Nat. Immunol. 10, 949-957 (2009).

36. Ha, E.M., Oh, C.T., Bae, Y.S. \& Lee, W.J. A direct role for dual oxidase in Drosophila gut immunity. Science 310, 847-850 (2005).

37. Ha, E.M. et al. An antioxidant system required for host protection against gut infection in Drosophila. Dev. Cell 8, 125-132 (2005).

38. Lee, K.A. et al. Bacterial-derived uracil as a modulator of mucosal immunity and gut-microbe homeostasis in Drosophila. Cell 153, 797-811 (2013).
39. Ferrandon, D. The complementary facets of epithelial host defenses in the genetic model organism Drosophila melanogaster: from resistance to resilience. Curr. Opin. Immunol. 25, 59-70 (2013).

40. Ahn, H.M., Lee, K.S., Lee, D.S. \& Yu, K. JNK/FOXO mediated PeroxiredoxinV expression regulates redox homeostasis during Drosophila melanogaster gut infection. Dev. Comp. Immunol. 38, 466-473 (2012).

41. Karpac, J., Biteau, B. \& Jasper, H. Misregulation of an adaptive metabolic response contributes to the age-related disruption of lipid homeostasis in Drosophila. Cell Rep. 4, 1250-1261 (2013).

42. Guo, L., Karpac, J., Tran, S.L. \& Jasper, H. PGRP-SC2 promotes gut immune homeostasis to limit commensal dysbiosis and extend lifespan. Cell 156, 109-122 (2014).

43. Nehme, N.T. et al. A model of bacterial intestinal infections in Drosophila melanogaster. PLoS Pathog. 3, e173 (2007).

44. Bosco-Drayon, V., Poidevin, M., Boneca, I.G., Narbonne-Reveau, K., Royet, J. \& Charroux, B. Peptidoglycan sensing by the receptor PGRP-LE in the Drosophila gut induces immune responses to infectious bacteria and tolerance to microbiota. Cell Host Microbe 12, 153-165 (2012).

45. Ragab, A., Buechling, T., Gesellchen, V., Spirohn, K., Boettcher, A.L. \& Boutros, M. Drosophila Ras/MAPK signalling regulates innate immune responses in immune and intestinal stem cells. EMBO J. 30, 1123-1136 (2011).

46. Cronin, S.J. et al. Genome-wide RNAi screen identifies genes involved in intestinal pathogenic bacterial infection. Science 325, 340-343 (2009).

47. Takehana, A. et al. Overexpression of a pattern-recognition receptor, peptidoglycan-recognition protein-LE, activates imd/relish-mediated antibacterial defense and the prophenoloxidase cascade in Drosophila larvae. Proc. Natl Acad. Sci. USA 99, 13705-13710 (2002).

48. Wagner, C., Isermann, K. \& Roeder, T. Infection induces a survival program and local remodeling in the airway epithelium of the fly. FASEB J. 23, 2045-2054 (2009).

49. Bettencourt, R., Asha, H., Dearolf, C. \& Ip, YT. Hemolymph-dependent and -independent responses in Drosophila immune tissue. J. Cell Biochem. 92, 849-863 (2004).

50. Hwangbo, D.S., Gershman, B., Tu, M.P., Palmer, M. \& Tatar, M. Drosophila $\mathrm{dFOXO}$ controls lifespan and regulates insulin signalling in brain and fat body. Nature 429, 562-566 (2004).

51. Ferrandon, D. et al. A drosomycin-GFP reporter transgene reveals a local immune response in Drosophila that is not dependent on the Toll pathway. EMBO J. 17, 1217-1227 (1998).

52. Junger, M.A. et al. The Drosophila forkhead transcription factor FOXO mediates the reduction in cell number associated with reduced insulin signaling. J. Biol. 2, 20 (2003).

53. Singh, S.R., Mishra, M.K., Kango-Singh, M. \& Hou, S.X. Generation and staining of intestinal stem cell lineage in adult midgut. Methods Mol. Biol. 879, 47-69 (2012).

54. Warmbold, C. et al. Dermatophagoides pteronyssinus major allergen 1 activates the innate immune response of the fruit fly Drosophila melanogaster. J. Immunol. 190, 366-371 (2013).

55. Pfaffl, M.W. A new mathematical model for relative quantification in realtime RT-PCR. Nucleic Acids Res. 29, e45 (2001). 